\title{
Quantitative Comparison between Differential Adhesion Models and Cell Sorting in the Presence and Absence of Fluctuations
}

\author{
José C. M. Mombach,* James A. Glazier, Richard C. Raphael, and Mark Zajac \\ Department of Physics, University of Notre Dame, Notre Dame, Indiana 46556
}

(Received 20 January 1995)

\begin{abstract}
We study the effect of inhibition of cell-membrane ruffling on cell sorting in experiments and simulations. We use aggregates of neural retinal and pigmented retinal cells obtained from chicken embryos and inhibit membrane ruffling by adding the drug cytochalasin B to the culture medium. Simulations using the large- $Q$ Potts model with differential adhesivity, with the simulation temperature controlling the membrane ruffling amplitude, agree quantitatively with the experimental results for both normal and low fluctuation sorting, providing strong support for the validity of the model. In both cases, inhibition of ruffling prevents large scale cell sorting.

PACS numbers: 87.45.Bp, 68.10.Cr, 75.10.Hk, 87.10.+e
\end{abstract}

While cell sorting was first observed qualitatively in 1744 [1], its kinetics have never been studied quantitatively. When cells obtained from different tissues of an embryo are dissociated and mixed to form aggregates, they sort to form coherent homotypic (like cell) domains (see Fig. 1). In some species of sponges, sea urchins, and hydras even a whole adult animal can completely regenerate [1].

This cell sorting is analogous to the demixing of imiscible liquids. For example, when water is dispersed in oil, energy minimization causes the water droplets to merge and form a single droplet. The surface tension of aggregates of cells obtained from the liver and heart of chick embryos have area-invariant properties characteristic of liquid behavior [2]. Steinberg proposed that differences in adhesion (surface tensions) between different types of cells [3] drive cell sorting. In this case, different energies are associated with different interfaces between cells: homotypic, heterotypic (unlike cells), and cell-medium boundaries. Minimizing the overall surface energy (as in liquids) drives the system to a final fixed configuration [4]. However, Steinberg's model does not clarify the mechanism by which surface energies drive the cells.

Membrane activity in cells seems to be an essential ingredient of complete cell sorting. Armstrong and Parenti have used cytochalasin B to inhibit cell membrane ruffling in aggregates [5]. They found that aggregates that normally sort, do not sort, or sort only partially in the presence of this drug [6]. However, their work was inconclusive since they did not determine to what degree cell sorting depends on membrane activity.

Large- $Q$ Potts model simulations [7] yield various patterns in two and three dimensions, including complete sorting and engulfment, showing that differential adhesivity and random membrane fluctuations are sufficient to explain simple cell sorting of random aggregates qualitatively, though in normal development many other mechanisms are important as well. Sorting presents two distinct phases: the formation of the external (light) layer charac- terized by fast cell motion, followed by a much slower bulk rearrangement (see Fig. 2).

In this work we present the first quantitative comparison between experiment and the Potts model simulation of cell sorting. We consider two different mechanisms that could guide cell rearrangement. (a) Passive: no fluctuation mechanism such as membrane activity is necessary and an aggregate of cells can spontaneously reach a pattern with global minimum energy. (b) Fluctuating: at least random membrane fluctuations are required to reach the minimum energy. If hypothesis (a) holds, then aggregates of cells with passive membranes should be able to sort completely due only to adhesion gradients; if not, then membrane fluctuations are necessary and (b) holds.

In our experiments we used neural retinal and pigmented retinal cells obtained from 9- to 10-day old chick embryos. Cells were isolated using standard techniques; see Ref. [6]. The ratios used ranged from 3 to 10 neural retinal cells for one pigmented retinal cell. We monitored aggregation by taking images at regular time intervals. After 9-15 h, when the external light layer was complete and the aggregates were compact, some aggregates were transferred to be cultured singly in $50 \mathrm{ml}$ culture flasks in a $37^{\circ} \mathrm{C}$ shaking water bath to observe their time evolution. For the low fluctuation case we began the experiment using a medium containing cytochalasin $\mathrm{B}$ at a concentration of $10 \mu \mathrm{g} / \mathrm{ml}$. Aggregates of normal size and composition form over normal time scales in the presence of cytochalasin $\mathrm{B}$, showing that the drug does not interfere with normal cell adhesion, regardless of the detailed chemistry; see Ref. [6].

Our simulation uses an extended large- $Q$ Potts model on a cubic $100^{3}$ lattice as described in Ref. [7]. In the model, when the fluctuation temperature $T$ increases cell boundaries fluctuate with greater amplitude, simulating membrane fluctuations of real cells (not to be confused with the small amplitude thermal fluctuations due to real temperature). To eliminate fragments we anneal briefly at $T=0$ before display and statistical characterization. 
(a)

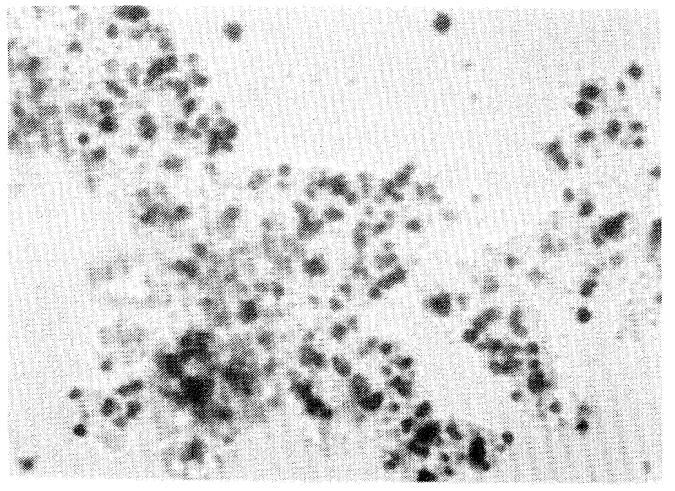

(b)

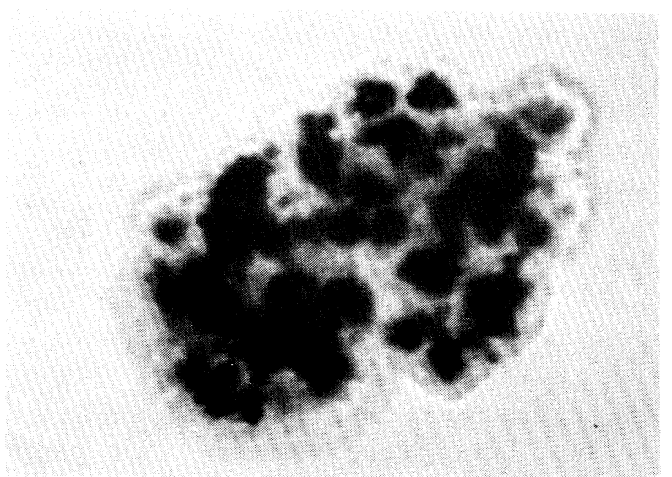

(c)

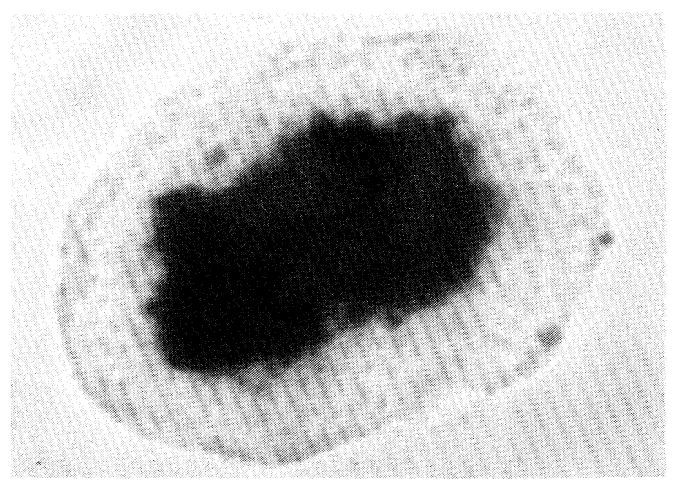

FIG. 1. Sorting of an aggregate of neural retinal (light) and pigmented retinal cells (dark) obtained from 10-day old chick embryos. (a) Aggregate $5 \mathrm{~h}$ after mixing of dissociated cells. (b) Aggregate after $10 \mathrm{~h}$. The neural retinal cells form an external layer. (c) Aggregate after $72 \mathrm{~h}$. The neural retinal cells surround an internal dark core of pigmented retinal cells.

The surface tensions are [7] $\sigma(l, l)=4.5$ for lightmedium interface, $\sigma(d, d)=7.0$ for dark-medium interface, and $\sigma(d, l)=0.5$ for dark-light interface which yields a ratio between light-medium and dark-medium surface tensions of 0.64 . The experimental surface tensions for liver and heart cell aggregates of chick embryos are $4.3 \pm 0.1$ and $8.3 \pm 0.1 \mathrm{dyn} / \mathrm{cm}$, respectively, which yield a ratio of 0.52 [2]. For ectodermal and endodermal cells of Hydra vulgaris this ratio is estimated to be

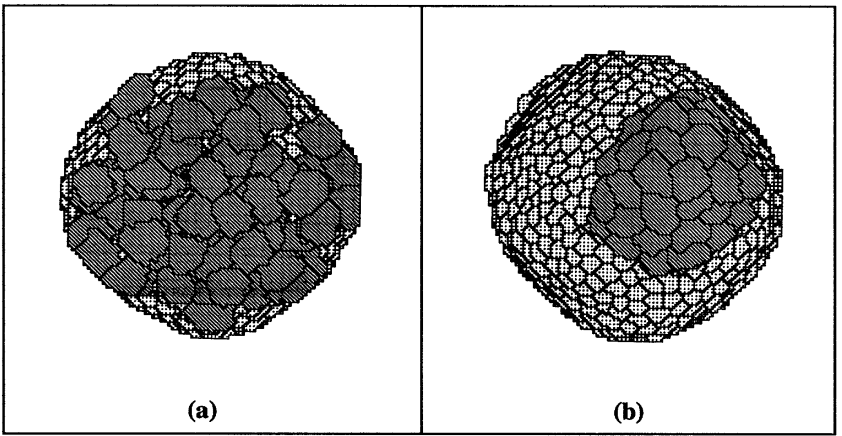

FIG. 2. Computer simulation of cell sorting using the Potts model with differential adhesivity $(T=32)$. (a) Initial round aggregate with randomly assigned cell types. A complete external light cell layer forms after $100 \mathrm{MCS}$ (not shown). (b) The aggregate sorts completely after $4000 \mathrm{MCS}$

0.6 [8]. Unfortunately, in these experiments the surface tensions in the heterotypic cell interface were not measured. However, we are constrained by the equation [2] $\sigma(d, d)>[\sigma(d, d)+\sigma(l, l) \sigma(d, l)] / 2 \geq \sigma(l, l)$.

Since fractional boundary lengths depend on the ratios and relative size of the different types of cells, we set the parameters in the simulation to the corresponding experimental values. The average diameter of almost spherical cells is $\sim 5 \mu \mathrm{m}( \pm 0.3 \mu \mathrm{m})$ for neural retinal cells and $9 \mu \mathrm{m}( \pm 1.6 \mu \mathrm{m})$ for pigmented retinal cells, which yield a volume ratio of $\sim 5.8$. Although cells are not spherical in the aggregates, the volume ratio still holds. In the simulation, the ratio of the target volumes of light and dark cells is set to 5.8 with a typical volume of light cells of 100 sites, and of dark cells, 580 sites. From completely sorted spheroidal aggregates we counted approximately 30 neural retinal cells for each pigmented retinal cell in the aggregates selected for study, which we employ in the simulation.

To simulate normal sorting we set the fluctuation temperature $T=32$, giving typical fluctuations of $\sim 1$ lattice site corresponding to experimentally observed fluctuations of about $1 \mu \mathrm{m}$ [9] for cells with a diameter of $5-10 \mu \mathrm{m}$. At $T=0$ the simulated aggregates do not evolve in time due to residual lattice anisotropy [7]. Since cell membranes fluctuate due to real temperature at an amplitude of roughly a tenth of the amplitude of normal cytoskeletally driven fluctuations [9], in the presence of cytochalasin B we set $T=4$.

The images obtained from the experimental aggregates are two-dimensional vertical projections of a threedimensional structure. In the simulation we project the aggregate onto a plane, by scanning the columns of the three-dimensional matrix. A column with a dark cell generates a dark site on the projected plane, a column composed only of light cells generates a light site, and only of medium generates a medium site. With this algorithm all dark cells in our simulated aggregates are visible. 
This approximation holds since these aggregates are small, $2 \times 10^{3}$ cells. The experimental aggregates have more cells, which hides some dark cells due to light dispersion, introducing a small error $(<10 \%)$ in our measured boundary length. This difference decreases in time to zero for aggregates that sort completely due to the elimination of isolated dark cells. However, we expect a small difference in our results for aggregates cultured in medium containing cytochalasin B since they contain isolated dark cells even at long times.

The aggregates we studied ranged in diameter from 150 to $300 \mu \mathrm{m}$ and the estimated number of cells from $2 \times 10^{4}$ to $1.6 \times 10^{5}$. The effect of cytochalasin $\mathrm{B}$ on sorting can be seen in Fig. 3(a). As found by Armstrong, sorting was partial. The aggregates were not rounded, a complete light layer and an internal dark core did not

(a)

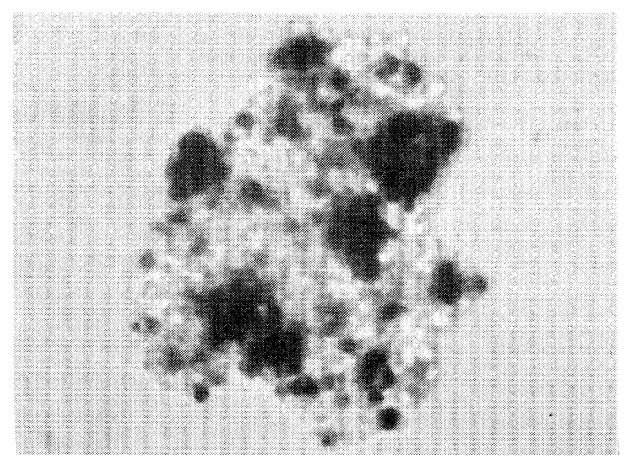

(b)

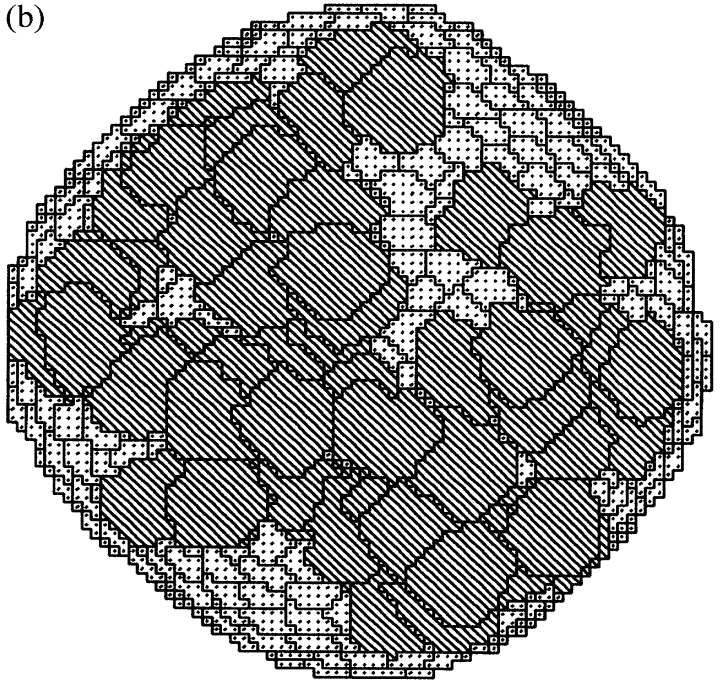

FIG. 3. Representative images of the experimental and simulated aggregates (two-dimensional projections of threedimensional aggregates) at long times. (a) Partially sorted aggregate of neural retinal and pigmented retinal cells of chick embryos cultured in medium containing cytochalasin B after 83 h. (b) Computer simulation of the effect of cytochalasin B using the Potts model with differential adhesivity at $4000 \mathrm{MCS}$ $(T=4)$. form, unlike normal sorting [see Fig. 1(c)]. Instead, some clusters of dark cells formed, presenting a local order very different from the global order found in the normal case. Aggregates transferred from medium containing cytochalasin B to be cultured in medium free of the drug, after $40 \mathrm{~h}$ recovered and sorted normally over normal time scales. When the transfer was made at later times, $60 \mathrm{~h}$ or more, sorting was not complete or did not occur, probably due to the decrease of membrane activity and stabilization of cells contacts, since both mechanisms decrease cell mobility [10]. We have confirmed experimentally that cell mobility decreases substantially around $60 \mathrm{~h}$ [11]. The simulation at $T=4$, Fig. 3(b), resembles the experiment. Sorting is also partial but no clusters of dark cells form because of the small number of cells in the simulated aggregate, compared to the experiment. We are currently running simulations with $2 \times 10^{4}$ cells in larger lattices from which we expect to obtain a better visual similarity to experimental aggregates [12].

At a fluctuation temperature of $T=32$, sorting is complete; see Fig. 2(b). In Fig. 4(a) and 4(b) we plot the time evolution of the boundaries between 5 and $87 \mathrm{~h}$ for partial and normal sorting, respectively. Points represent the average of 2-6 different aggregates. The time in the simulation was rescaled to real time in the experiment by the best coincidence between order parameters at

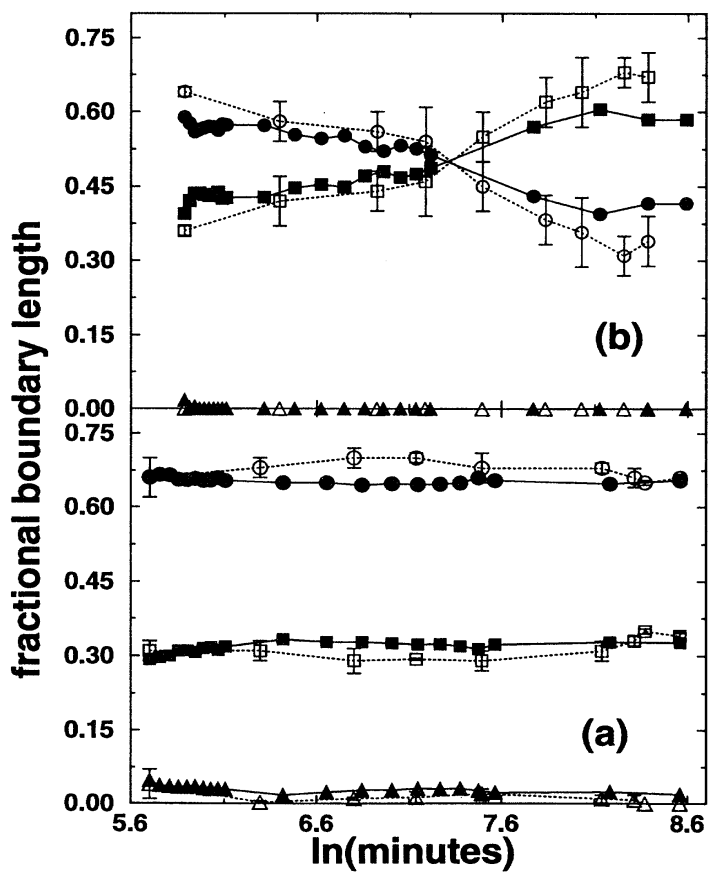

FIG. 4. Time evolution of boundary lengths for partial (a) and complete (b) cell sorting for experiment (open symbols) and simulation (closed symbols). Circles, boundary between dark and light cells. Squares, boundary between light cells and medium. Triangles, boundary between dark cells and medium. Error bars are 1 standard deviation. 
early and late stages. In partial sorting the dark-medium boundary quickly decreases in the initial $4 \mathrm{~h}$ and then becomes constant as a partial light cell layer forms. The light-medium and dark-light boundaries increase in the initial $4 \mathrm{~h}$ due to the decrease of the dark-medium boundary and then remain constant. In normal sorting the dark-light boundary decreases as both cell types segregate and the light-medium boundary increases while the darkmedium boundary decreases, as a result of the formation of the light cell layer, until they reach plateaus. The formation of the external light cell layer is much faster than the characteristic time of the whole sorting process. We attribute the faster saturation of the boundaries for the simulated aggregate to its size. We expect to improve the quantitative agreement by simulating larger aggregates.

Inhibition of membrane ruffling would also prevent cells from following diffusible chemical gradients by chemotaxis. Thus, our cytochalasin $\mathrm{B}$ results do not by themselves rule out chemotaxis. We have performed experiments using a few isolated dark cells in a large light cell aggregate. The motion of the individual dark cells is random, and not influenced by the presence of nearby (13 cell diameters) dark cells. If chemotaxis were important we would expect the dark cells to cluster even at low concentration [11].

The logarithmic time scale of sorting results from the cells overcoming increasingly higher energy barriers and from the decreased mobility of larger cell clusters. Neither effect operates in chemotaxis in which each cell follows a gradient independently, and in which gradient strength increases as cluster size grows, hence its power law time scales.

Our results suggest an energy landscape with moderate local minima, where the fluctuations of cell membranes play a role analogous to temperature in ordinary materials, increasing the mobility of cells by allowing them to interact with neighbors. As a consequence, the system can overcome energy barriers and explore ergodically the energy space to reach its global minimum. Despite thermal fluctuations, it does not seem possible for cells lacking a locomotive apparatus to sort completely due to adhesion gradients [13] [hypothesis (a) above], since energy minima tend to stop the evolution. Engulfment, in which the light and dark cells are brought together as coherent tissues and whose final state is identical to that of cell sorting, should have much smaller energy barriers. We would expect that engulfment would proceed normally, though more slowly, regardless of the presence of cytochalasin B. We are currently checking this hypothesis experimentally.

Aspects of cell sorting to be investigated to improve the realism of the model include the length scale and amplitude of cell membrane fluctuations and better measurement of adhesivity of cells and cell-medium surface tensions in real aggregates. We are currently measuring the fluctuation amplitude and spectrum. Initial results indicate that the cytoskeletal fluctuations are quasithermal in spectrum [11]. The simulation is being improved to describe more complex biological behavior like the early stages of an embryo (blastulation) by increasing the number of different types of cells and introducing mitosis [14].

We thank C.R. Mohr and J. M. Clark for their help with experiments, and the partial support of Ford Motor Company, Exxon Educational Foundation, ACS/PRF, NSF/NYI DMR-9257011, and the Brazilian Agency CNPq.

*Permanent address: Instituto de Física, Universidade Federal do Rio Grande do Sul, C.P. 15051, 91501-970 Porto Alegre, RS, Brazil. Electronic address: jose@if1.if.ufrgs.br

[1] S. G. Lenhoff, Hydra and the Birth of Experimental Biology-1744 (The Boxwood Press, Pacific Grove, 1986); P.L. Townes and J. Holtfreter, J. Exp. Zool. 128, 53 (1955); P. B. Armstrong, Crit. Rev. Biochem. Mol. Biol. 24, 119-149 (1989); S. A. Newman and W. D. Comper, Development 110, 1 (1990).

[2] R. A. Foty, G. Forgacs, C. M. Pfleger, and M. S. Steinberg, Phys. Rev. Lett. 72, 2298 (1994).

[3] Cells adhere because they have molecules on their surface specialized in adhesion; M. Takeichi, Science 251, 1451 (1991). Neural retinal cells express neural cell adhesion molecules among other cell adhesion molecules; A.F. Williams and A. N. Barclay, Annu. Rev. Immunol. 6, 381 (1988).

[4] M.S. Steinberg, Science 141, 401 (1963).

[5] Cytochalasin B depolymerizes the cellular network of microfilaments of actin (the cytoskeleton) responsible for membrane ruffling.

[6] P. B. Armstrong and D. Parenti, J. Cell Biol. 55, 606 (1972). Aggregates of the same size, shape and cell type distribution form in the same time in the presence or absence of cytochalasin B.

[7] F. Graner and J. A. Glazier, Phys. Rev. Lett. 69, 2013 (1992); J. A. Glazier and F. Graner, Phys. Rev. E 47, 2128 (1993); J. A. Glazier, R. C. Raphael, F. Graner, and Y. Sawada, in Interplay of Genetic and Physical Processes in the Development of Biological Form, edited by D. Beysens, M.-A. Felix, G. Forgacs and F. Gaill (Springer, New York, to be published).

[8] M. Sato-Maeda, M. Uchida, F. Graner, and H. Tashiro, Dev. Biol. 162, 77 (1994).

[9] F. Graner (private communication).

[10] We have also reduced membrane fluctuations by holding cells at $4{ }^{\circ} \mathrm{C}$, resulting in partial cell sorting identical to that seen with cytochalasin $\mathrm{B}$. If we return the cells to $37{ }^{\circ} \mathrm{C}$ we obtain complete sorting over normal time scales, indicating that the cells are not significantly damaged by refrigeration.

[11] J. C. M. Mombach and J. A. Glazier (to be published).

[12] J.C. M. Mombach et al. (to be published).

[13] F. Graner and Y. Sawada, J. Theor. Biol. 164, 477 (1993).

[14] J. C. M. Mombach, R. M. C. de Almeida, and J. R. Iglesias, Phys. Rev. E 48, 598 (1993). 
(a)

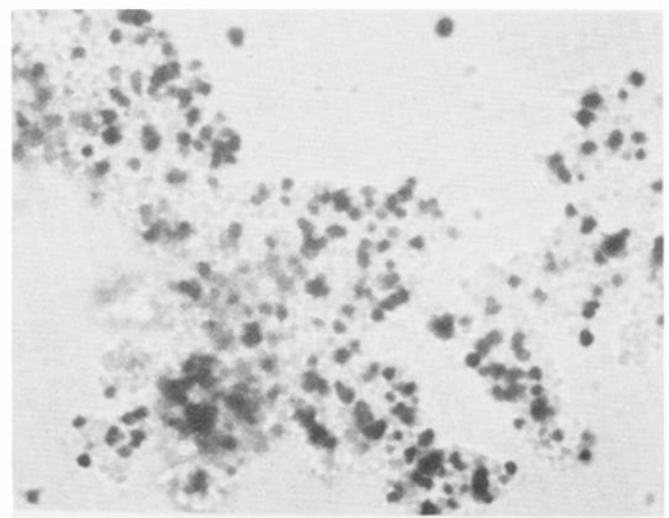

(b)

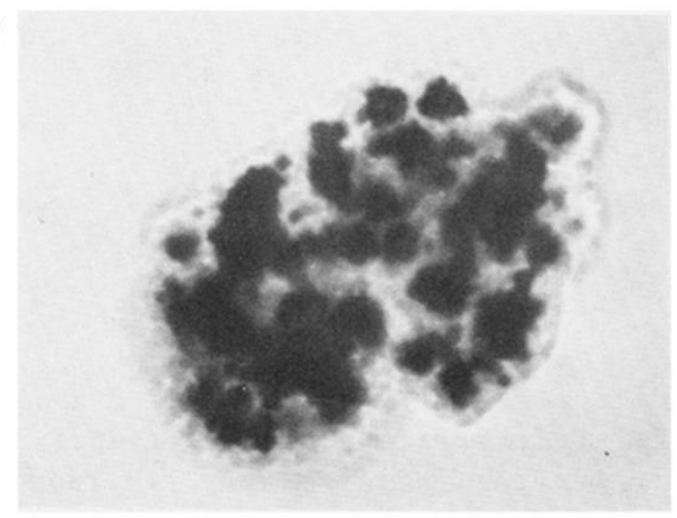

(c)

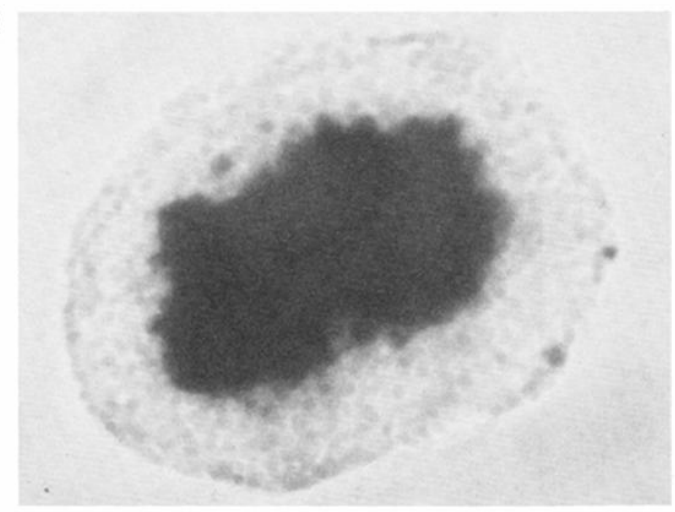

FIG. 1. Sorting of an aggregate of neural retinal (light) and pigmented retinal cells (dark) obtained from 10-day old chick embryos. (a) Aggregate $5 \mathrm{~h}$ after mixing of dissociated cells. (b) Aggregate after $10 \mathrm{~h}$. The neural retinal cells form an external layer. (c) Aggregate after $72 \mathrm{~h}$. The neural retinal cells surround an internal dark core of pigmented retinal cells. 
(a)

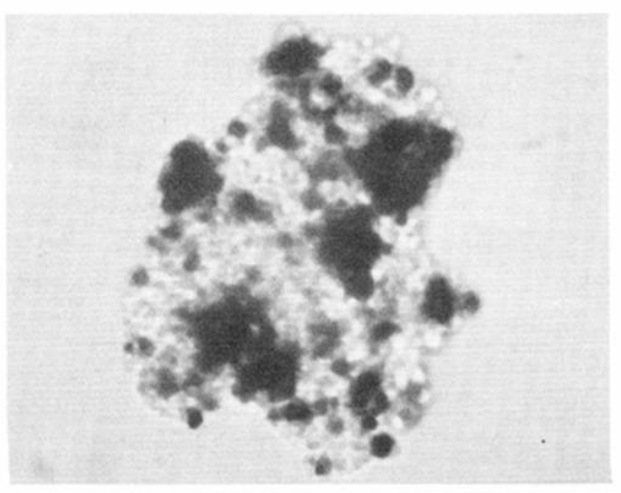

(b)
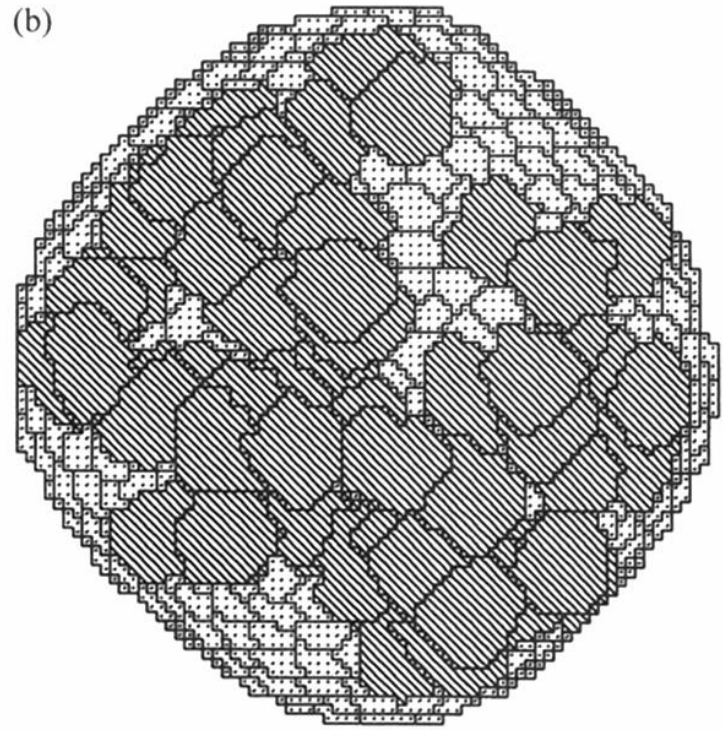

FIG. 3. Representative images of the experimental and simulated aggregates (two-dimensional projections of threedimensional aggregates) at long times. (a) Partially sorted aggregate of neural retinal and pigmented retinal cells of chick embryos cultured in medium containing cytochalasin B after 83 h. (b) Computer simulation of the effect of cytochalasin B using the Potts model with differential adhesivity at $4000 \mathrm{MCS}$ $(T=4)$. 